\title{
Hot-Tail Runaway Seed Landscape during the Thermal Quench in Tokamaks
}

\author{
Ida Svenningsson $\odot,{ }^{1}$ Ola Embreus, ${ }^{1}$ Mathias Hoppe $\odot,{ }^{1}$ Sarah L. Newton, ${ }^{2}$ and Tünde Fülöp $\odot^{1}$ \\ ${ }^{1}$ Department of Physics, Chalmers University of Technology, SE-41296 Gothenburg, Sweden \\ ${ }^{2}$ Culham Centre for Fusion Energy, Abingdon, Oxon OX14 3DB, United Kingdom
}

(Received 13 January 2021; revised 28 May 2021; accepted 2 June 2021; published 14 July 2021)

\begin{abstract}
Runaway electron populations seeded from the hot tail generated by the rapid cooling in plasmaterminating disruptions are a serious concern for next-step tokamak devices such as ITER. Here, we present a comprehensive treatment of the thermal quench, including the superthermal electron dynamics, heat and particle transport, atomic physics, and radial losses due to magnetic perturbations: processes that are strongly linked and essential for the evaluation of the runaway seed in disruptions mitigated by material injection. We identify limits on the injected impurity density and magnetic perturbation level for which the runaway seed current is acceptable without excessive thermal energy being lost to the wall via particle impact. The consistent modeling of generation and losses shows that runaway beams tend to form near the edge of the plasma, where they could be deconfined via external perturbations.
\end{abstract}

DOI: 10.1103/PhysRevLett.127.035001

One of the crucial problems facing magnetic fusion devices with large plasma currents is the occurrence of plasma-terminating disruptions [1]. Such events can result in a partial loss of magnetic confinement and a sudden cooling of the plasma. This thermal quench (TQ) is associated with an increase in the plasma resistivity and impedes current flow. As a result, a strong inductive electric field arises, which can lead to runaway acceleration of electrons to relativistic energies [2-6].

Predictions show that a large part of the initial plasma current in ITER, and other high-current tokamaks, can thus be converted to a beam of energetic electrons $[7,8]$. The current carried by a "seed" of runaway electrons is greatly amplified in an avalanche process, with a multiplication factor exponentially sensitive to the initial plasma current [2]. The subsequent uncontrolled loss of these electrons could lead to localized power deposition and damage of the plasma facing components (PFCs) [9].

The proposed disruption mitigation method in ITER is massive material injection, using a combination of deuterium and impurities $[10,11]$. When injected in a predisruptive plasma, the impurity atoms can radiate away the stored thermal and magnetic energy, while the deuterium raises the critical electric field required for runaway. The mitigation system should be such that it provides sufficient radiation to reduce conductive losses during the TQ, to avoid damage on PFCs [9].

Published by the American Physical Society under the terms of the Creative Commons Attribution 4.0 International license. Further distribution of this work must maintain attribution to the author(s) and the published article's title, journal citation, and DOI. Funded by Bibsam.
Material injection creates a two-component electron distribution, consisting of hot electrons from the original bulk Maxwellian population and cold electrons ionized from the injected material $[3,4,12]$. The hot electrons lose energy due to the interaction with the cold population, but in rapidly cooling plasmas, due to the low collision frequency for fast electrons, a "hot tail" often remains in the electron distribution [13]. Hot-tail generation is efficient in the early phase of the disruption, the TQ, when the temperature changes by orders of magnitude; in ITER, from tens of keVs to a few eVs in a millisecond. This hottail seed can be the dominant source of primary runaway electrons [14-17], yet remains poorly modeled. The topology of the magnetic field confining the particles also changes and stochastic regions are formed, allowing rapid radial transport of runaway electrons [18-20]. Therefore, a large part of the hot-tail seed is expected to be deconfined.

Recent numerical studies indicate that assuming all hottail electrons remain in the plasma overestimates the final runaway current in experiments at ASDEX Upgrade $[21,22]$. However, differences, for example, due to size and initial plasma current, may be expected between disruption scenarios which can be studied on existing machines and those which would occur in ITER. Reliable predictions of the hot-tail seed generation during the rapid TQ, in particular, accounting for the deconfinement of seed electrons, is needed to determine the runaway conversion in disruptions. Limitation of the runaway population by fluctuations during the subsequent current quench phase (dominated by avalanching) has been studied previously $[23,24]$. Self-consistent modeling of the transport, power balance, and runaway generation is essential also in the TQ, as these effects influence the TQ dynamics 
and the associated induced electric field, which in turn drives the runaway generation.

Here we present an integrated model of thermal quench dynamics, including hot-tail generation and losses due to magnetic perturbations, and use it to explore viable scenarios with combined deuterium and neon injection. We model the current evolution together with the magnetic field fluctuation induced energy and particle transport, as the injected material and bulk plasma evolve into a cold free electron population and hot population, with densities $n_{\text {cold }}$ and $n_{\text {hot }}$, respectively.

The total current density is given by the sum of Ohmic, hot electron, and runaway current densities: $j_{\|}=E \sigma_{\text {cold }}+\int_{|\boldsymbol{p}|<p_{\mathrm{c}}} e v_{\|} f d \boldsymbol{p}+j_{\mathrm{RE}}$, where $\sigma_{\text {cold }}$ is the Spitzer conductivity, $v_{\|}$is the velocity parallel to the magnetic field, $f$ is the superthermal (hot) electron distribution function, $p_{\mathrm{c}}$ the critical runaway momentum [25] and the runaway current is defined as $j_{\mathrm{RE}}=\int_{p_{\mathrm{c}}<|\boldsymbol{p}|<p_{\max }} e v_{\|} f d \boldsymbol{p}+e c n_{\mathrm{RE}}$. Here, $n_{\mathrm{RE}}$ is the density of electrons having momentum $p>p_{\max }$ and $E$ is the electric field parallel to the magnetic field, which in the cylindrical approximation evolves at radius $r$ according to

$$
\mu_{0} \frac{\partial j_{\|}}{\partial t}=\frac{1}{r} \frac{\partial}{\partial r}\left(r \frac{\partial E}{\partial r}\right)
$$

The temperature of the cold electron population is determined from the associated energy density $W_{\text {cold }}=$ $(3 / 2) n_{\text {cold }} T_{\text {cold }}$, which is evolved according to

$$
\begin{aligned}
\frac{\partial W_{\text {cold }}}{\partial t}= & \sigma_{\text {cold }} E^{2}-n_{\text {cold }} \sum_{i} \sum_{j=0}^{Z_{i}-1} n_{i}^{(j)} L_{i}^{(j)}\left(T_{\text {cold }}, n_{\text {cold }}\right) \\
& +Q_{c}+\frac{1}{r} \frac{\partial}{\partial r}\left[r D_{W} \frac{\partial T_{\text {cold }}}{\partial r}\right],
\end{aligned}
$$

where $n_{i}^{(j)}$ is the number density of an ion with atomic number $Z_{i}$ and charge number $Z_{0 j}=j$, and $L_{i}^{(j)}$ are the line radiation rates. The heat diffusion coefficient $D_{W}$ is calculated by integrating the kinetic radial diffusion coefficient $D$ over a Maxwellian corresponding to the cold electron population: $D_{W}=n_{\text {cold }} /\left(\pi^{3 / 2} m_{e}^{3} v_{T}^{3} T_{\text {cold }}\right)$ $\int\left(m_{e} v^{2} / 2\right)\left(v^{2} / v_{T}^{2}-3 / 2\right) D(\boldsymbol{v}) \exp \left(-v^{2} / v_{T}^{2}\right) d \boldsymbol{p}, \quad$ with $v_{T}=\sqrt{2 T_{\text {cold }} / m_{e}}$ as the thermal velocity of the cold electron population. The rate of collisional energy transfer $Q_{c}=\int \Delta \dot{E}_{e e} f d \mathbf{p}+\sum_{i} Q_{e i}$, where $\int \Delta \dot{E}_{e e} f d p$ is the energy transfer from the hot electrons to cold free electrons, with $\Delta \dot{E}_{e e}=4 \pi n_{\text {cold }} r_{0}^{2} \ln \Lambda_{e e} m_{e} c^{4} / v, r_{0}$ is the classical electron radius, and the sum is taken over ion species. The rate of collisional energy transfer between two Maxwellians is denoted $Q_{k l}=\left\langle n Z^{2}\right\rangle_{k}\left\langle n Z^{2}\right\rangle_{l} e^{4} \ln \Lambda_{k l}$ $\left[(2 \pi)^{3 / 2} \epsilon_{0}^{2} m_{k} m_{l}\right]^{-1}\left(T_{k}-T_{l}\right)\left(T_{k} / m_{k}+T_{l} / m_{l}\right)^{-3 / 2}$, with $\left\langle n Z^{2}\right\rangle_{k}=\sum_{j=0}^{Z_{k}-1} Z_{0 j}^{2} n_{k}^{(j)}$ and the ion temperatures are evolved according to $(3 / 2) \partial\left(\langle n Z\rangle_{i} T_{i}\right) / \partial t=\sum_{j} Q_{i j}$.

The time evolution of the impurity species is described by $\partial n_{i}^{(j)} / \partial t=I_{i}^{(j-1)} n_{i}^{(j-1)} n_{\text {cold }}-I_{i}^{(j)} n_{i}^{(j)} n_{\text {cold }}+$ $R_{i}^{(j+1)} n_{i}^{(j+1)} n_{\text {cold }}-R_{i}^{(j)} n_{i}^{(j)} n_{\text {cold }}$, where $I_{i}^{(j)}$ include the ionization rate and electron-impact ionization coefficients with the cold electrons, and $R_{i}^{(j)}$ are radiative recombination rates, obtained from OpenADAS [26]. The number density of cold electrons $n_{\text {cold }}$ is such that the overall plasma is charge neutral, satisfying $\sum_{i} \sum_{j} Z_{0 j} n_{i}^{(j)}=n_{\text {cold }}+n_{\mathrm{RE}}+\int f d \boldsymbol{p}$ at all radii.

The superthermal electron dynamics is determined by the kinetic equation, which in radius, momentum, and pitch coordinates $(r, p, \xi)$, where $v_{\|}=v \xi$, reads

$$
\begin{aligned}
\frac{\partial f}{\partial t}+ & e E\left(\frac{1}{p^{2}} \frac{\partial}{\partial p}\left[p^{2} \xi f\right]+\frac{1}{p} \frac{\partial}{\partial \xi}\left[\left(1-\xi^{2}\right) f\right]\right) \\
= & \frac{1}{p^{2}} \frac{\partial}{\partial p}\left[p^{3} \nu_{s} f\right]+\frac{\nu_{D}}{2} \frac{\partial}{\partial \xi}\left[\left(1-\xi^{2}\right) \frac{\partial f}{\partial \xi}\right] \\
& +\frac{1}{r} \frac{\partial}{\partial r}\left[r D \frac{\partial f}{\partial r}\right],
\end{aligned}
$$

where we neglect the energy-diffusion term, an assumption strictly valid only in the superthermal limit. Here $\nu_{s}(p)$ and $\nu_{D}(p)$ are the slowing-down and deflection frequencies due to particle collisions [27].

The runaway density $n_{\mathrm{RE}}$ evolves according to

$$
\frac{\partial n_{\mathrm{RE}}}{\partial t}=F_{p}\left(p_{\max }\right)+\frac{1}{r} \frac{\partial}{\partial r}\left(r D \frac{\partial n_{\mathrm{RE}}}{\partial r}\right),
$$

where the flux from the superthermal region into the runaway region is $F_{p}=2 \pi p^{2} \int\left(e E \xi-p \nu_{s}\right) f d \xi$, integrated along the upper boundary of the domain representing the kinetic hot electrons, $p_{\max }=3 m_{e} c$. As we focus on the generation of the hot-tail seed during the initial phase of the disruption (the TQ), we do not include here the runaway growth due to avalanche multiplication, which occurs on a longer timescale.

We have taken the Rechester-Rosenbluth form for the coefficient $D=\pi q v_{\|} R(\delta B / B)^{2}$ [28] for simplicity, which assumes that the magnetic field is fully stochastic. Here, $R$ is the major radius of the tokamak, $\pi q R$ represents the parallel scale length of the magnetic perturbation, and $\delta B / B$ is the normalized magnetic fluctuation amplitude, where in this Letter we assume the fluctuating field amplitude to be constant in space and time. Although such an assumption is not typical in the TQ, our results provide a bound on the perturbation level necessary to remove the hot-tail seed entirely. The precise details of the transport coefficient $D$ are relatively unimportant to the present study; we present scans over its magnitude and are mainly 
concerned with its relative importance to the heat transport and not the electron evolution in any specific magnetic field configuration.

The plasma model detailed here unifies and extends components that have appeared in previous studies. Atomic screening effects [27] and fast-electron transport [3] extend the kinetic equation beyond that in [12]. The electric field and current evolution has been widely used, e.g., in Refs. [17,25], but only recently in kinetic simulations [29]. The thermal energy equation is fully charge-state resolved, as in the KPRAD model [30] or Ref. [25], and captures electron heat transport from field perturbations [28] and nonthermal electron heating [12].

In the following, we solve the coupled equations (1)-(4) with the DREAM code (Disruption Runaway Electron Analysis Model), with full capabilities described in [31], and present results for an ITER-like disruption with a combined deuterium and neon injection. The initial plasma is fully ionized deuterium with a predisruption electron density profile assumed to be uniform with a value of $n_{\text {hot }}=10^{20} \mathrm{~m}^{-3}$. (Due to the limited appearance of the ion mass, the 50/50 deuterium-tritium composition does not significantly alter the results.) The initial temperature profile of the electron and deuterium populations is given by $T(r)=15\left[1-(r / a)^{4}\right] \mathrm{keV}$. The initial current density profile is assumed to be $j_{\|}(r)=j_{0}\left[1-(r / a)^{4}\right]^{3 / 2}$, with $j_{0}$ chosen to give a total plasma current $I_{p}$. The major and minor radii are $R=6.2 \mathrm{~m}$ and $a=2 \mathrm{~m}$. Neutral neon and deuterium are introduced with a prescribed density profile and zero temperature at the start of the simulation.

In order to assess the operational space leading to acceptable hot-tail generation, in Fig. 1 we present scans over injected impurity density and perturbation level, using the Rechester-Rosenbluth transport model with a radially constant magnetic perturbation (taking $q \approx 1$ ). Each point in the plane corresponds to a TQ simulation, with constant prescribed profiles of total impurity density and $\delta B / B$. Colors indicate the maximum runaway current reached during the simulation. To avoid significant avalanche generation, the runaway seed current must be lower than $10 \mathrm{~A}$ in the $8 \mathrm{MA}$ case and $10^{-4} \mathrm{~A}$ in the $15 \mathrm{MA}$ case [25]. These limits are indicated (for dominant hot-tail seed) with solid lines. To avoid damage to the first wall, at least $90 \%$ of the thermal energy loss must come from radiation [9]. This gives an upper limit on how strong the transport can be, which has been indicated with a dashed line under the assumption that all kinetic energy transported through the edge of the plasma will strike the wall.

Figure 1 shows that there is a region in parameter space with moderate injected impurity density and radial transport (between the solid and dashed lines), which gives acceptable hot-tail generation and nonradiative heat transport to the first wall. Simulations with only neon injection (not shown) indicate a very restricted acceptable operating

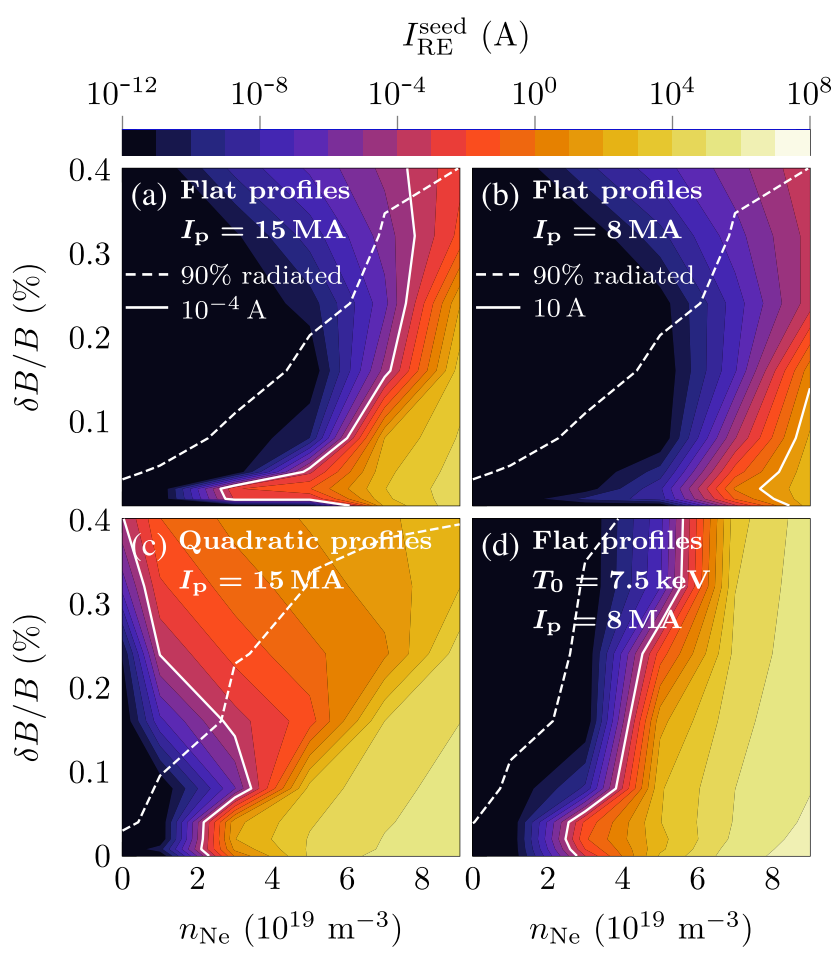

FIG. 1. Maximum seed runaway current as a function of injected neon density and normalized magnetic perturbation $\delta B / B$. The injected deuterium density is $n_{D}=10^{21} \mathrm{~m}^{-3}$. Injected deuterium and neon profiles assumed to be (a),(b),(d) flat and (c) quadratic $n_{\mathrm{Ne} / \mathrm{D}}(r)=n_{\mathrm{Ne} / \mathrm{D}}\left[2 / 11+(18 / 11)(r / a)^{2}\right]$. Below the dashed lines, the transport losses are acceptably low, and left of the solid lines the hot-tail seed is acceptably small. The initial central temperature is $T_{0}=15 \mathrm{keV}$ in (a),(b),(c).

space, due to a lower radiated fraction. For lower initial plasma current, the parameter region widens, as shown in Fig. 1(b); i.e., greater impurity injection can be allowed, mainly due to the increased acceptable seed currents.

Nonuniform impurity deposition profiles are likely to arise in realistic material injection scenarios. They can reduce the heat transported to the wall, as radiation losses scale quadratically with plasma density, while transport scales only linearly. Therefore, in Fig. 1(c) we investigate the effect of density profiles taking the form $n_{\mathrm{Ne} / \mathrm{D}}(r)=n_{\mathrm{Ne} / \mathrm{D}}\left[2 / 11+(18 / 11)(r / a)^{2}\right]$, chosen so the edge impurity density is 10 times higher than that at the center, and the total number of ions corresponds to flat profiles at the constant values $n_{\mathrm{Ne}}$ and $n_{\mathrm{D}}$. This quadratic profile moderately widens the parameter region of tolerable transport losses (see the shift in the dashed line), however, a significant increase in runaway current is observed, especially in the high- $\delta B / B$ region. This increase of the runaway current due to transport is caused by the low density in the center of the plasma, allowing fast electrons to persist due to the lower collisionality. When these core electrons are transported to the cool edge-where electric fields are stronger-they can be accelerated as runaways. 
Figure 1(d) illustrates the case with a lower initial temperature, in which case hot-tail generation is more efficient [cf. Fig. 1(b)]. This counterintuitive result agrees with earlier findings [12] and is because, in disruptions dominated by radiation losses, the TQ time decreases faster with temperature than the collision time. Thus, strong electric fields capable of accelerating runaways are generated before the hot tail has had time to slow down.

To understand in detail the basic dynamics of a TQ triggered by material injection, we show the evolution of temperature, electric field and current in Fig. 2. The Figs. 2 (a), 2(b) show the cold electron temperature and induced electric field at different radial positions. In the Figs. 2(c)2(f), we compare the effect of radial transport on the radial evolution of the cold electron temperature and hot and runaway electron current densities. In Figs. 2(c) and 2(e) no radial transport was assumed $(D=0)$, and in Figs. 2(d) and 2(f) $\delta B / B=0.16 \%$. According to magnetohydrodynamic (MHD) simulations of disruptions induced by material injection in the Joint European Torus tokamak, the normalized perturbation amplitude during the TQ can be around $\delta B / B \simeq 1 \%$ or higher [19]. The choice $\delta B / B=0.16 \%$ is a conservative estimate, giving a characteristic transport time of runaways $t_{d}=a^{2} / D=a^{2} /\left[\pi c R(\delta B / B)^{2}\right] \approx 0.3 \mathrm{~ms}$. The energy confinement time is a few milliseconds, which is of the same order of magnitude as the estimated TQ time in ITER [6].

The initial electron distribution function is a Maxwellian, carrying the initial density and temperature. The injected material is ionized by the interaction with the hot plasma, cools the plasma due to line radiation, and provides additional background electrons that are initially cold, but are heated via collisional energy exchange with the hot population, which quickly slows down as the plasma cools due to radiation. At low temperatures, Ohmic heating and radiation losses dominate, and when in balance they support a stable equilibrium temperature typically in the 5$20 \mathrm{eV}$ range. In such regions, the electric field tends to be strong, allowing efficient runaway acceleration and, as a consequence, radially localized current sheets may arise $[16,32,33]$. Near the plasma edge, due to the lower current density and initial temperature, the background electrons enter the cold equilibrium near $5 \mathrm{eV}$ at the beginning of the TQ. Meanwhile, in the core of the plasma, Ohmic heating and energy transfer from hot electrons can heat the background to hundreds of $\mathrm{eV}$, which can be sustained for multiple milliseconds before the cold equilibrium is ultimately reached. A strong electric field is then induced, as illustrated in Fig. 2(b). This occurs first at the edge and propagates inward as the cold front reaches the central parts of the plasma.

Figures 2(e) and 2(f) show the radial evolution of the plasma current. The initial current carried by hot electrons is rapidly converted to Ohmic current carried by the cold background plasma as the hot electrons are slowed down by collisions. The hot-tail electrons that are accelerated by the electric field and enter the runaway region are shown with solid lines. Radial transport of electrons in stochastic fields causes the central temperature to reach the cold equilibrium point at an earlier time, causing an earlier onset of strong electric fields and subsequent increased runaway acceleration. Note that the first runaway acceleration occurs at the outer radii, which might lead to hollow runaway density profiles. The reduction in the maximum runaway current density $j_{\mathrm{RE}}$ in Fig. 2(f) compared to Fig. 2(e) is due to the transport by magnetic perturbations. This indicates that inducing strong edge fluctuations can be useful in mitigating the runaway population produced by the strong edge cooling.

The simulations also show that the maximum runaway current varies nonmonotonically with magnetic perturbation $\delta B / B$. This is illustrated in Fig. 3, where we show examples of the energy density $W_{\text {cold }}+W_{\text {hot }}$ and current evolution for three different values of $\delta B / B$, where $W_{\text {hot }}=\int(\gamma-1) m_{e} c^{2} f d \mathbf{p}$. With the two lower values, $\delta B / B=0.04 \%$ (blue) and $0.16 \%$ (red), the runaway current (solid) peaks at similar times, but since more hot particles remain in the plasma when $\delta B / B=0.04 \%$, this case results in a higher peak value. The highest value $\delta B / B=0.4 \%$ results in the shortest TQ [yellow line in
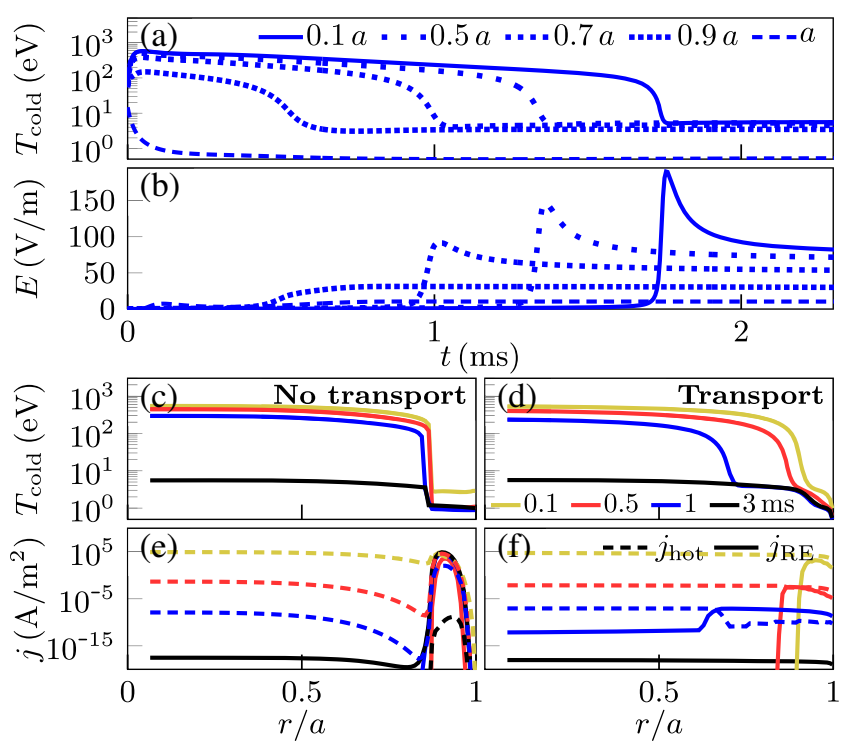

FIG. 2. Simulation of an ITER-like disruption with initial plasma current $I_{p}=15 \mathrm{MA}$ and injected deuterium and neon densities $n_{\mathrm{D}}=10^{21} \mathrm{~m}^{-3}$ and $n_{\mathrm{Ne}}=9 \times 10^{19} \mathrm{~m}^{-3}$, respectively. Injected deuterium and neon profiles assumed to be flat and in (a), (b) $\delta B / B=0.16 \%$. (a) Cold electron temperature as a function of time at five different radii. (b) Induced electric field at the same radial positions as in (a). (c),(d) Cold electron temperature as a function of radius at four different times. (e),(f) Hot (dashed) and runaway (solid) current density profiles at the same times as in (c),(d). (c),(e) $\delta B / B=0$. (d),(f) $\delta B / B=0.16 \%$. 


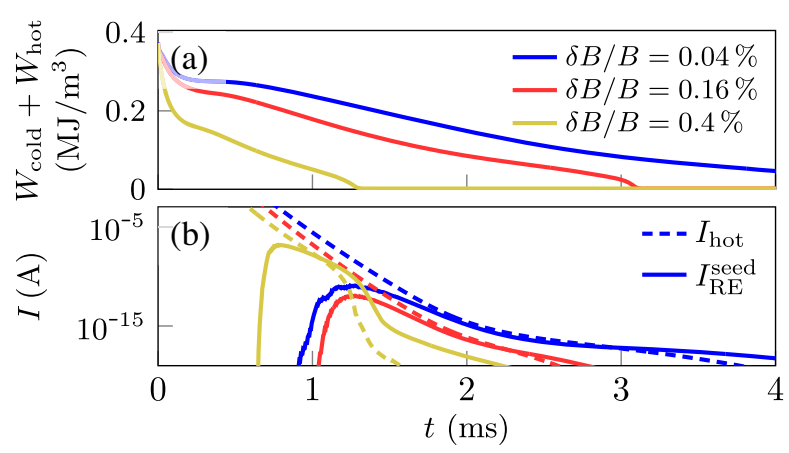

FIG. 3. (a) Electron energy density and (b) current for $\delta B / B=$ $0.04 \%$ (blue), $\delta B / B=0.16 \%$ (red), and $\delta B / B=0.4 \%$ (yellow) and injected densities $n_{\mathrm{D}}=10^{21} \mathrm{~m}^{-3}, n_{\mathrm{Ne}}=5 \times 10^{19} \mathrm{~m}^{-3}$.

Fig. 3(a)], which causes an earlier rise in the electric field and earlier runaway acceleration. Previous predictions have suggested that the hot-tail seed would decrease monotonically with TQ duration [34]. Our results indicate that the interplay between fast-electron transport and temperature evolution must be considered carefully in order to predict the seed current.

Reduced MHD simulations [18] indicate natural disruption activity gives fluctuations peaked in the core. Such radially varying magnetic perturbations give quantitative differences, reducing the acceptable parameter space, but the hot-tail landscape is similar. As expected, the runaway seed is larger if the $\delta B / B$ radial profile is peaked at the core. Optimizing the net profile by externally applied perturbations, exploring transport coefficients motivated by the MHD simulations, provides a potential route to identify robust operation spaces.

In this Letter, we assumed instantaneous deposition of the injected material, valid when the deposition timescale is much shorter than the timescale of plasma cooling, which is often the case in pellet injection experiments in mediumsized devices [35]. When the timescales are comparable, which is the case for larger devices such as ITER (traversing a $2 \mathrm{~m}$ radius in $2 \mathrm{~ms}$ requires a $1 \mathrm{~km} / \mathrm{s}$ pellet speed, much higher than the few hundreds of $\mathrm{m} / \mathrm{s}$ foreseen [36]), the model presented here should be complemented by an impurity injection model, such as pellet ablation. The resulting nonuniform cooling as the pellet crosses the plasma has implications for the limits on impurity density and perturbation level, as we have illustrated with the nonuniform deposition profile. However, in those cases where alarming runaway currents are predicted, they tend to form near the edge of the plasma, where they can more easily be deconfined by, e.g., external perturbations [24,37] during the current quench. A potentially important effect not addressed here is the MHD stability of such current profiles, which might lead to safe termination [38].

In summary, our results show that, in the case of moderate impurity and deuterium injection, radial transport caused by magnetic fluctuations during the TQ should allow for efficient losses of hot-tail seed runaways in ITERlike disruptions, without producing excessive heat loads on the first wall. The cutoff value of impurity density below which the runaway current is acceptable depends on the material deposition profile and the prequench temperature, as well as the possibility of exciting magnetic perturbations.

The authors are grateful to E. Nardon, I. Pusztai, G. Papp, and the rest of the Plasma Theory group for fruitful discussions. This work was supported by the European Research Council (ERC) under the European Unions Horizon 2020 Research and Innovation Programme (ERC-2014-CoG Grant No. 647121) and the Swedish Research Council (Dnr. 2018-03911).

[1] T. Hender, J. Wesley, J. Bialek, A. Bondeson, and A. Boozer (The ITPA MHD, Disruption \& Magnet Group) et al., Nucl. Fusion 47, S128 (2007).

[2] M. Rosenbluth and S. Putvinski, Nucl. Fusion 37, 1355 (1997).

[3] S. Chiu, M. Rosenbluth, R. Harvey, and V. Chan, Nucl. Fusion 38, 1711 (1998).

[4] R. W. Harvey, V. S. Chan, S. C. Chiu, T. E. Evans, M. N. Rosenbluth, and D. G. Whyte, Phys. Plasmas 7, 4590 (2000).

[5] A. H. Boozer, Phys. Plasmas 22, 032504 (2015).

[6] B. N. Breizman, P. Aleynikov, E. M. Hollmann, and M. Lehnen, Nucl. Fusion 59, 083001 (2019).

[7] A. H. Boozer, Nucl. Fusion 58, 036006 (2018).

[8] R. Sweeney, A. J. Creely, J. Doody, T. Fülöp, D. T. Garnier, R. Granetz, M. Greenwald, L. Hesslow, J. Irby, V. A. Izzo, R. J. L. Haye, N. C. Logan, K. Montes, C. Paz-Soldan, C. Rea, R. A. Tinguely, O. Vallhagen, and J. Zhu, J. Plasma Phys. 86, 865860507 (2020).

[9] M. Lehnen, K. Aleynikova, P. Aleynikov, D. Campbell, P. Drewelow et al., J. Nucl. Mater. 463, 39 (2015).

[10] E. M. Hollmann, P. B. Aleynikov, T. Fülöp, D. A. Humphreys, V. A. Izzo, M. Lehnen, V. E. Lukash, G. Papp, G. Pautasso, F. Saint-Laurent, and J. A. Snipes, Phys. Plasmas 22, 021802 (2015).

[11] E. Nardon, D. Hu, M. Hoelzl, and D. Bonfiglio (The JOREK team), Nucl. Fusion 60, 126040 (2020).

[12] P. Aleynikov and B. N. Breizman, Nucl. Fusion 57, 046009 (2017).

[13] P. Helander, H. Smith, T. Fülöp, and L.-G. Eriksson, Phys. Plasmas 11, 5704 (2004).

[14] H. Smith, P. Helander, L.-G. Eriksson, and T. Fülöp, Phys. Plasmas 12, 122505 (2005).

[15] H. M. Smith and E. Verwichte, Phys. Plasmas 15, 072502 (2008).

[16] T. Fehér, H. M. Smith, T. Fülöp, and K. Gál, Plasma Phys. Controlled Fusion 53, 035014 (2011).

[17] J. Martín-Solís, A. Loarte, and M. Lehnen, Nucl. Fusion 57, 066025 (2017).

[18] V. Izzo, E. Hollmann, A. James, J. Yu, D. Humphreys, L. Lao, P. Parks, P. Sieck, J. Wesley, R. Granetz, G. Olynyk, and D. Whyte, Nucl. Fusion 51, 063032 (2011). 
[19] K. Särkimäki, O. Embreus, E. Nardon, and T. Fülöp (JET contributors), Nucl. Fusion 60, 126050 (2020).

[20] D. Hu, E. Nardon, M. Hoelzl, F. Wieschollek, M. Lehnen, G. Huijsmans, D. C. van Vugt, and S.-H. Kim (JET contributors, and JOREK team), Nucl. Fusion 61, 026015 (2021).

[21] K. Insulander Björk, O. Vallhagen, G. Papp, O. Embréus, E. Rachlew, and T. Fülöp (ASDEX Upgrade Team, the JET contributors, and the EUROfusion MST1 Team), Plasma Phys. Controlled Fusion 63, 085021 (2021).

[22] M. Hoppe, L. Hesslow, O. Embreus, L. Unnerfelt, G. Papp, I. Pusztai, T. Fülöp, O. Lexell, T. Lunt, and E. Macusova (The ASDEX Upgrade team, and the EUROfusion MST1 team), J. Plasma Phys. 87, 855870102 (2021).

[23] P. Helander, L.-G. Eriksson, and F. Andersson, Phys. Plasmas 7, 4106 (2000).

[24] P. Svensson, O. Embreus, S. L. Newton, K. Särkimäki, O. Vallhagen, and T. Fülöp, J. Plasma Phys. 87, 905870207 (2021).

[25] O. Vallhagen, O. Embreus, I. Pusztai, L. Hesslow, and T. Fülöp, J. Plasma Phys. 86, 475860401 (2020).

[26] H. P. Summers, The ADAS user manual, version 2.6, http:// www.adas.ac.uk (2004).

[27] L. Hesslow, O. Embréus, M. Hoppe, T. DuBois, G. Papp, M. Rahm, and T. Fülöp, J. Plasma Phys. 84, 905840605 (2018).

[28] A. B. Rechester and M. N. Rosenbluth, Phys. Rev. Lett. 40, 38 (1978).

[29] R. Harvey, Y. V. Petrov, C. C. Kim, C. Forest, L. Lao, and P. Parks, Nucl. Fusion 59, 106046 (2019).
[30] D. Whyte and T. Jernigan, Energy balance, radiation and stability during rapid plasma termination via impurity pellet injections on DIII-D, Technical Report, General Atomics, San Diego, CA, U.S.; Oak Ridge National Lab., TN, 1997.

[31] M. Hoppe, O. Embreus, and T. Fülöp, arXiv:2103.16457 [Comp. Phys. Commun. (to be published)].

[32] S. Putvinski, N. Fujisawa, D. Post, N. Putvinskaya, M. Rosenbluth, and J. Wesley, J. Nucl. Mater. 241-243, 316 (1997), https://www.sciencedirect.com/science/article/abs/ pii/S0022311597800566?via\%3Dihub.

[33] L.-G. Eriksson, P. Helander, F. Andersson, D. Anderson, and M. Lisak, Phys. Rev. Lett. 92, 205004 (2004).

[34] H. M. Smith, T. Fehér, T. Fülöp, K. Gál, and E. Verwichte, Plasma Phys. Controlled Fusion 51, 124008 (2009).

[35] C. Paz-Soldan, P. Aleynikov, E. Hollmann, A. Lvovskiy, I. Bykov, X. Du, N. Eidietis, and D. Shiraki, Nucl. Fusion 60, 056020 (2020).

[36] L. Baylor, C. C. Barbier, J. R. Carmichael, S. K. Combs, M. N. Ericson, N. D. B. Ezell, P. W. Fisher, M. S. Lyttle, S. J. Meitner, D. A. Rasmussen, S. F. Smith, J. B. Wilgen, S. Maruyama, and G. Kiss, Fusion Sci. Technol. 68, 211 (2015), https://www.tandfonline.com/doi/abs/10.13182/ FST14-926.

[37] H. M. Smith, A. H. Boozer, and P. Helander, Phys. Plasmas 20, 072505 (2013).

[38] C. Reux, C. Paz-Soldan, P. Aleynikov, V. Bandaru, O. Ficker, S. Silburn, M. Hoelzl, S. Jachmich, N. Eidietis, M. Lehnen, and S. Sridhar (JET contributors), Phys. Rev. Lett. 126, 175001 (2021). 